\title{
Microenvironmental considerations in the application of human mesenchymal stem cells in regenerative therapies
}

\author{
Steven J Greco' \\ Pranela Rameshwar ${ }^{2}$ \\ 'Graduate School of Biomedical \\ Sciences; ${ }^{2}$ Department of Medicine, \\ Division of Hematology/Oncology, \\ University of Medicine and Dentistry \\ of New Jersey-New Jersey Medical \\ School, Newark, NJ, USA
}

\begin{abstract}
The therapeutic utilization of stem cells has been ongoing for several decades, principally in the form of bone marrow (BM) transplants to treat various hematological disorders and other immune-related diseases. More recently, stem cells have been examined as a potential therapy for a multitude of other diseases and disorders, many of which are currently untreatable. One consideration that poses a formidable task for the successful clinical application of stem cells in new disease models is the impact of the host tissue microenvironment on the desired therapeutic outcome. In vitro, stem cells exist in surroundings directly controllable by the researcher to produce the desired cellular behavior. In vivo, the transplanted cells are exposed to a dynamic host microenvironment laden with soluble mediators and immunoreactive cells. In this review, we focus on the possible contribution by microenvironmental factors, and how these influences can be overcome in therapies utilizing mesenchymal stem cells (MSCs), such as for graft versus host disease, multiple sclerosis and ischemia among others. Specifically, we examine three ubiquitous microenvironmental factors, IL- $1 \alpha / \beta$, TNF $\alpha$, and SDF- $1 \alpha$, and consider how inhibitors and receptor antagonists to these molecules could be applied to increase the efficacy of MSC therapies while minimizing unforeseen harm to the patient.
\end{abstract}

Keywords: cytokine, microenvironment, mesenchymal stem cells, bone marrow

\section{Introduction}

The emergence of stem cells as a therapeutic for many diseases, disorders and injuries has brought excitement among scientists, clinicians and patients alike regarding the potential treatment of previously untreatable conditions. However, the implementation of many stem cell therapies in patients may still be years away. When considering translating these therapies into patients, there are two principal concerns that must be resolved: I. Can the stem cells efficiently produce the desired therapeutic outcome, albeit tissue replacement or repair, in vitro?; and II. Can the in vitro studies be replicated in vivo, both short- and long-term, with increased confidence? Much of the past research has concentrated on question one, or more appropriately, the philosophy of can we apply the method? However, to recognize stem cells as key factors in the treatment of various ailments, we need to rest assured that we can also answer question two - Is this a viable treatment approach? These questions are aside from the ethical implications surrounding the field, which ask should we do it.

In any case, stem cells will continue to be researched as a potential treatment for a multitude of diseases and disorders. Considerable progress has been made in addressing the first question stated above - can we do it. A vast number of tissue types have been generated from both embryonic (ES cells) and adult stem cells. ES cells are pluripotent cells derived from the inner cell mass of the blastocyst, which hold tremendous potential in generating specified tissue types (Lerou and Daley 2005). 
However, the potential for immune rejection, together with the possibility of tumor formation has caused their application in humans to proceed with caution (Lerou and Daley 2005). Adult stem cells tend to be tissue-specific cells with limited differentiation potential compared with ES cells. Adult stem cells are clinically attractive therapies due to their reduced risk of tumorigenesis and ability to expand with relative ease (Cheng et al 2004).

Among the many types of adult stem cells, those resident to the bone marrow (BM), particularly mesenchymal stem cells (MSCs), have gained extensive interest among scientists and clinicians (Deans and Moseley 2000). MSCs are mesodermal cells primarily resident to the adult BM, which undergo lineage-specific differentiation to generate bone, fat, and cartilage among other tissue types (Bianco et al 2001). MSCs have also been reported to transdifferentiate into defined ectodermal and endodermal tissues in vitro, thus alluding to their inherent plasticity (Choi and Panayi 2001; Cho et al 2005; Eberhardt et al 2006; Ong et al 2006; Greco and Liu et al 2007; Greco and Zhou et al 2007; Jeon et al 2007; Trzaska et al 2007). MSCs are available for autologous therapies, have a unique ability to bypass immune rejection and are inherently migratory (Potian et al 2003). These properties of MSCs make them particularly well suited when considering the second question posed earlier - can in vitro findings be accurately recapitulated in vivo? Whereas tissues derived from ES cells or other types of stem cells may be rejected when transplanted, MSCs offer the potential for allogeneic transplantation and a readily available source of "off-the-shelf" stem cells for personalized therapies.

However, the unique immune properties of MSCs do not guarantee that the cells will produce the desired therapeutic outcome or even that they will not be rejected. In vitro, a MSC's growth conditions can be closely monitored to favor stem cell growth and/or differentiation. In vivo, the transplanted MSCs are exposed to local immune cells and soluble mediators that could influence the cells' behavior, either positively or negatively regarding the desired outcome. This concept of the tissue microenvironment has become a growing concern among researchers, and may be the ultimate factor in deciding whether a stem cell therapy succeeds or fails (Greco et al 2004; Yan et al 2006; Grassel and Ahmed 2007; Phinney and Prockop 2007).

A prototypical example of a tissue microenvironment affecting stem cell behavior is observed among hematopoietic stem cells (HSCs) and their niche within the BM. HSCs are relatively quiescent cells located close to the BM endosteum at relatively low oxygen concentration (Greco et al 2004).
As HSCs differentiate, the maturing immune cells migrate towards the central sinus of the BM under progressively higher oxygen concentrations (Greco et al 2004). The change in oxygen is a key determinant in the maturation of the immune cells before they leave the BM and migrate into the peripheral circulation (Greco et al 2004). In contrast, MSCs are located close to trabecular bone near the central sinus of the BM (Bianco et al 2001). As MSCs migrate towards the endosteum under progressively lower concentrations of oxygen, the stem cells differentiate into stromal fibroblasts, which form the principal support structure for immune cell maturation (Bianco et al 2001).

This example demonstrates that local microenvironmental changes in variables such as oxygen concentration can drastically affect the behavior of MSCs. Since MSCs have been shown to generate a vast number of tissues, they have clinical implications in a wide array of diseases and disorders. Among possible transplantation sites are tissues such as cardiac, neural, pancreatic and bone. Each tissue provides a unique local microenvironment that can affect the success of the therapy. The problem facing researchers is accurately developing in vitro models to recapitulate the tissue microenvironment so that cellular behavior can be observed prior to transplantation. This is no easy task considering the dynamic nature of the microenvironment.

Transplantation of MSCs alone will generate a local immune response and disrupt homeostasis within the tissue milieu by causing release of inflammatory mediators, such as cytokines. The anatomy of the BM is such that MSCs are in direct interaction with immune cells and form synapse-like structures with innervating nerve fibers (Bianco et al 2001). MSCs express receptors for many cytokines and neurotransmitters, thus demonstrating their potential to respond to local microenvironmental changes (Greco and Rameshwar 2007). Excessive cytokine release within the transplantation site could lead to the production of other soluble factors by the MSCs themselves. If these factors are immunoreactive, then other immune cells could infiltrate the tissue and cause an exacerbated immune response, rejection of the transplant or differentiation of the MSCs (Figure 1A). On the other hand, MSCs have been shown to be a potent source of trophic factors (Phinney and Prockop 2007). These findings indicate that MSCs could also be used to aid normal tissue repair, perhaps even more so than in cell replacement. Whether transplanted MSCs cause an immune insult or help repair injured tissues may be difficult to determine unless appropriate models are developed to better predict the outcome. However, if MSCs are found to negatively impact the host 
microenvironment through exposure to soluble mediators, there are still potential methods to develop effective therapeutics. When considering the example presented in Figure 1A, inclusion of specific cytokine receptor antagonists or inhibitors could suppress the untoward effects of the host microenvironment on the transplanted MSCs, thus leading to defined therapeutic outcomes (Figure 1B). Throughout the remainder of this review, we will address the feasibility of using similar pharmacologic approaches in MSC transplants, while focusing on three ubiquitous microenvironmental factors: IL- $1 \alpha / \beta$, TNF $\alpha$, and SDF- $1 \alpha$. Specifically, we will examine how receptor antagonists or inhibitors to these factors, whether federally approved or in development, may limit the potential negative influences of the tissue microenvironment.

\section{Interleukin- I $\alpha / \beta$}

IL- $1 \alpha$ and IL- $1 \beta$ are members of the IL- 1 superfamily of cytokines. These pro-inflammatory mediators are primarily synthesized by macrophages, monocytes and dendritic cells, and are responsible for immune defense against infection (Table 1) (Dinarello 1994). IL-1 $\alpha$ and IL-1 $\beta$ are also key regulators of hematopoesis and the inflammatory process (Table 1) (Dinarello 1994). Both cytokines are found throughout the body, thus they are expected to be present within the local microenvironment of most tissues.

Our laboratory has previously demonstrated that MSCs express IL-1RI, which is the principal receptor for both IL- $\alpha$ and IL-1 $\beta$ (Greco and Rameshwar 2007). Interestingly, membrane expression of the receptor was maintained throughout the course of transdifferentiation of MSCs into functional neurons (Greco and Rameshwar 2007). If IL-1 $\alpha$ or IL-1 $\beta$ were found to have negative effects on MSCs, then these effects may also be seen on transplantable tissues differentiated from MSCs. These results have implications regarding the ideal stage of stem cell implantation, whether undifferentiated, partly differentiated or fully differentiated.

IL-1 $\alpha$ was found to alter the behavior of undifferentiated MSCs and neurons differentiated from MSCs (Greco and Rameshwar 2007). Specifically, stimulation of MSCs
A.

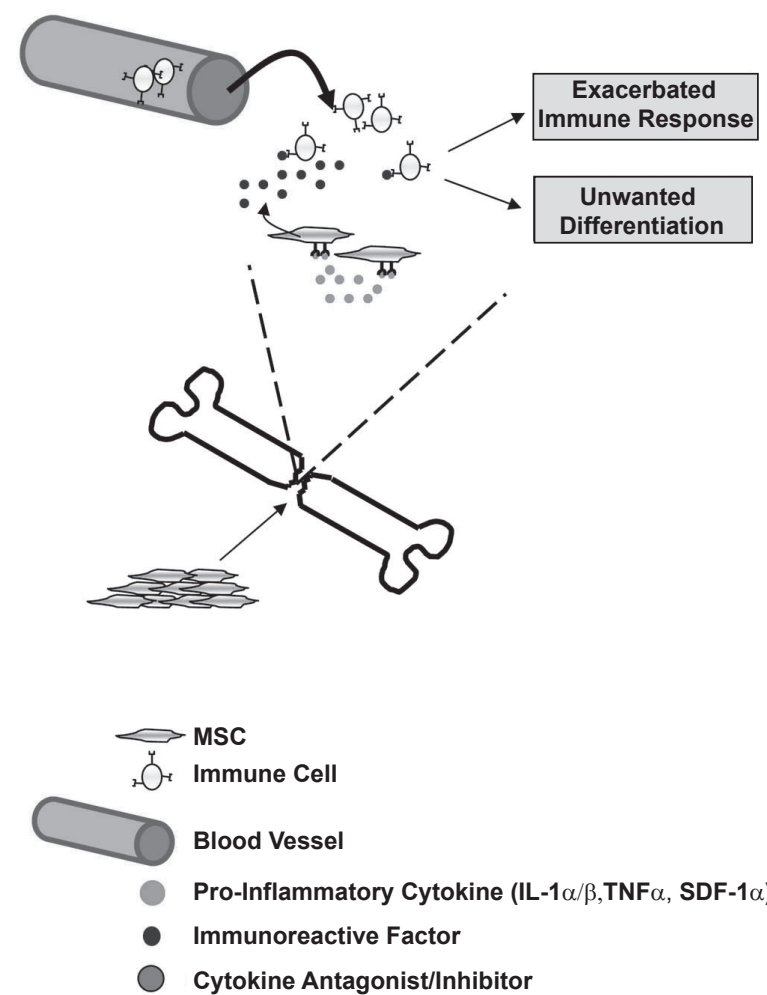

B.

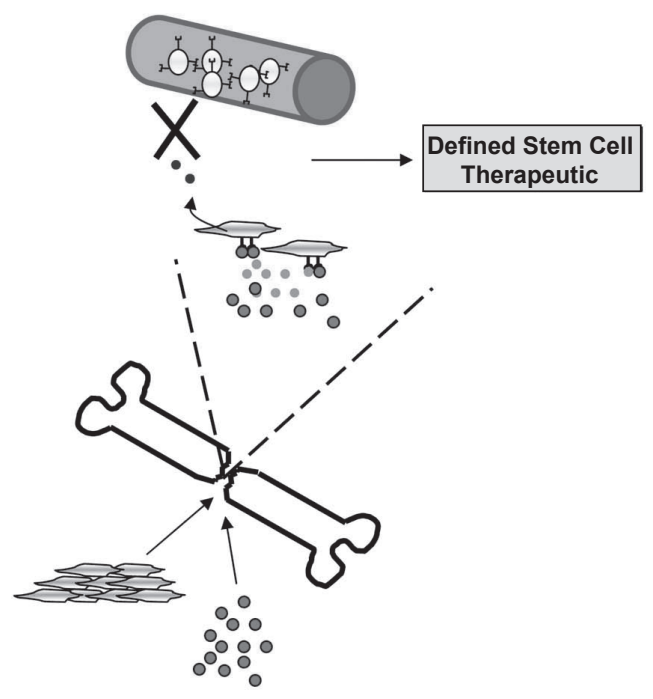

Figure I Cartoon depicting the potential effects of an inflammatory microenvironment on mesenchymal stem cell (MSC) therapies. A. An injured tissue, such as a bone fracture, is laden with inflammatory cytokines, such as IL-I $\alpha$, IL-I $\beta, T N F \alpha$, or IL-6, within the microenvironment. MSCs introduced into the microenvironment can respond to the inflammatory stimuli by synthesizing and releasing immunoreactive factors, such as substance P (SP) or other neuropeptides. Excessive production of SP would lead to an exacerbated immune response and infiltration of additional immune cells into the injured tissue.Additionally, the inflammatory stimuli could have an untoward effect on MSC differentiation, for example differentiation into fibroblasts rather than osteoblasts. B. Alternatively, delivery of MSCs together with an inflammatory cytokine antagonist/inhibitor may abrogate immunoreactivity and allow the desired stem cell therapeutic to proceed unhindered. 
Table I Microenvironmental factors implicated in the outcome of MSC therapies

\begin{tabular}{|c|c|c|c|c|c|}
\hline $\begin{array}{l}\text { Cytokine/ } \\
\text { chemokine }\end{array}$ & Source & $\begin{array}{l}\text { Physiological } \\
\text { function }\end{array}$ & $\begin{array}{l}\text { Receptor } \\
\text { expression } \\
\text { on MSCs }\end{array}$ & $\begin{array}{l}\text { Inhibitors/ } \\
\text { antagonists }\end{array}$ & Reference \\
\hline $\mathrm{IL}-\mathrm{I} \alpha / \beta$ & $\begin{array}{l}\text { macrophages, } \\
\text { monocytes, } \\
\text { dendritic cells }\end{array}$ & $\begin{array}{l}\text { immune response, } \\
\text { inflammation, } \\
\text { hematopoiesis }\end{array}$ & yes & IL-I ra, Kineret ${ }^{\circledR}$ & $\begin{array}{l}\text { Bresnihan and Cunnane } \\
\text { 1998; Gabay 2000; Arend } \\
\text { 1993; Hannum et al } 1990\end{array}$ \\
\hline $\mathrm{TNF} \alpha$ & macrophages & $\begin{array}{l}\text { immune response, } \\
\text { inflammation, } \\
\text { proliferation, } \\
\text { differentiation, } \\
\text { tumorigenesis, } \\
\text { viral replication }\end{array}$ & yes & $\begin{array}{l}\text { Remicade }^{\circledast}, \\
\text { Humira }^{\circledR}, \text { Enbrel }^{\circledR}\end{array}$ & $\begin{array}{l}\text { Knight et al 1993; Choy } \\
\text { and Panayi 200I }\end{array}$ \\
\hline SDF-I $\alpha$ & $\begin{array}{l}\text { tissue-specific } \\
\text { stromal cells }\end{array}$ & $\begin{array}{l}\text { immune response, } \\
\text { inflammation, } \\
\text { hematopoiesis, } \\
\text { chemotaxis, } \\
\text { tumor metastasis }\end{array}$ & yes & $\begin{array}{l}\text { Mozobil }^{\circledR}, \text { TI } 34, \\
\text { tannic acid }\end{array}$ & $\begin{array}{l}\text { Cashen et al 2007; } \\
\text { Arakaki et al 1999; Chen } \\
\text { et al } 2003\end{array}$ \\
\hline
\end{tabular}

Notes: Listed microenvironmental factors are ubiquitously expressed throughout the body and have known effects on mesenchymal stem cells MSCs. Co-therapy with pharmacologics, such as receptor antagonists or specific inhibitors, may improve the desired therapeutic outcome.

with IL- $1 \alpha$ caused production of the neurotransmitter, substance P (SP), by undifferentiated and differentiated cells (Greco and Rameshwar 2007). Similar effects were not observed in cells stimulated with IL-1 $\beta$ (Greco and Rameshwar 2007). SP has involvement in various physiological functions, such as the perception of pain and breast cancer progression, however the peptide also has a stimulatory effect on immune cell development and function (Greco et al 2004). SP was also found to stabilize IL-1RI mRNA, thus potentially forming an autocrine feedback loop whereby IL-1 $\alpha$ present in the microenvironment continually stimulates production of SP by the MSCs or their differentiated progeny (Greco and Rameshwar 2007). The excessive levels of SP could lead to immune cell infiltration and an exacerbated immune response, which may cause rejection of the transplant.

Interestingly, IL-1 $\alpha$ also had a more global effect on the behavior of MSCs, specifically their ability to transdifferentiate into neurons (Greco and Rameshwar 2007). MSCs that were grown in neuronal induction media containing IL-1 $\alpha$ showed greater expression of genes linked to neurogenesis compared to cells induced without IL-1 $\alpha$ (Greco and Rameshwar 2007). In comparison to the deleterious effects of IL-1 $\alpha$ mentioned above, these results demonstrate what appears to be a positive effect on MSC differentiation. If the desired therapeutic value of MSCs is transdifferentiation, in this case into neurons, then this finding can be considered beneficial. However, in many cases, the maintenance of MSCs as stem cells will be desired. Hence, the influence of the microenvironment on premature MSC differentiation would be unwarranted.

To counter the negative effects of IL- $1 \alpha$ present within a tissue microenvironment, co-therapies utilizing MSCs and specific IL-1R antagonists or inhibitors may be successful. The IL-1R antagonist (IL-1ra) is naturally occurring and binds to the IL-1RI. IL-1ra competes for binding to the IL-1RI with IL- $1 \alpha$ and IL- $1 \beta$, however binding of this ligand does not result in an intracellular signal (Dinarello 1994). A commercially available IL-1RI antagonist is Kineret ${ }^{\circledR}$, also known as Anakinra ${ }^{\circledR}$, which is a recombinant, non-glycosolated version of human IL-1 ra (Table 1) (Hannum et al 1990; Arend 1993; Bresnihan and Cunnane 1998; Gabay 2000). The drug has been used in the treatment of inflammatory conditions such as rheumatoid arthritis.

Recent studies in mice have shown that MSCs have an inherent ability to counteract the deleterious inflammatory effects of IL-1 $\alpha$ in injured tissues (Ortiz et al 2007). In response to bleomycin-induced inflammation and fibrosis within the lungs of mice, transplanted MSCs were shown to synthesize IL-1 ra to neutralize microenvironmental IL- $1 \alpha$. In addition, MSCs also protected the tissue from further damage by inhibiting the production of TNF $\alpha$ within the lung. If, however, exogenous IL-1ra supplementation is also necessary, it may be possible to administer an IL-1ra, such as Anakinra ${ }^{\circledR}$, during MSC transplantation. However, the means of accurately delivering the drug to its target is unknown. Perhaps the drug could be bound to the MSCs 
in such a way that the stem cells actually "piggy-back" the drug to the target. A great deal of research is still necessary to develop these types of therapeutics.

\section{Tumor necrosis factor $\alpha$}

TNF $\alpha$ is a pro-inflammatory cytokine principally synthesized by macrophages, which is involved in the acute phase of systemic inflammation (Locksley et al 2001). More specifically, TNF $\alpha$ mediates immune cell homing, proliferation and differentiation, as well as tumorigenesis and viral replication (Table 1) (Locksley et al 2001). TNF $\alpha$ is ubiquitously found throughout the body, and is another important factor present within local tissue microenvironments.

We have previously demonstrated that MSCs express the principal receptor for TNFo, TNF-R1 (Table 1) (Greco and Rameshwar 2007). Similar to IL-1RI expression, TNF-RI levels were maintained during the entire course of MSC transdifferentiation into neurons (Greco and Rameshwar 2007). However, whereas IL- $1 \alpha$ was able to induce SP production in undifferentiated, partly differentiated and fully differentiated cells, TNF $\alpha$ had similar effects only in partly and fully differentiated cells (Greco and Rameshwar 2007). Additionally, TNF $\alpha$ did not have the same enhancing effect on MSC transdifferentiation as IL-1 $\alpha$. These results re-emphasize that the ideal stage of differentiation for efficient transplantation is unknown. In the case of exposure to microenvironmental TNF $\alpha$, little to no negative effects may be observed with undifferentiated MSCs, while an exacerbated immune response may be seen if transplanting partly or fully transdifferentiated cells.

Recent studies have shown that undifferentiated MSCs incubated with TNF $\alpha$ have a greater ability to migrate in the presence of chemokines compared to cells incubated without TNF $\alpha$ (Ponte et al 2007; Schmal et al 2007). Increased chemotaxis of MSCs would be clinically important if proper homing to the site of tissue injury became more efficient. However, since MSCs are inherently chemotactic, increased sensitivity to chemokine gradients could cause continuous mobilization within a tissue, and impede proper homing and delivery of the desired therapeutic.

To offset any deleterious effects of microenvironmental TNF $\alpha$ on proper homing of MSCs to the site of tissue injury, co-thereapies with existing TNF $\alpha$ inhibitors may be beneficial. Currently there are three approved TNF $\alpha$ inhibitors, infliximab (Remicade ${ }^{\circledR}$ ), adalimumab (Humira ${ }^{\circledR}$ ) and etanercept $\left(\right.$ Enbrel $\left.^{\circledR}\right)$, which are primarily used to treat inflammatory and autoimmune disorders (Table 1)
(Knight et al 1993; Choy and Panayi 2001). Infliximab and adalimumab are monoclonal antibodies that bind TNF $\alpha$ and block signaling through the TNF-RI. Etanercept is a large molecular weight, soluble recombinant $\mathrm{TNF} \alpha$ receptor fusion protein that binds TNF $\alpha$ and prevents signaling through membrane-bound TNF-RI. Administration of these pharmacologics in combination with MSC therapies may negate any untoward effects of TNF $\alpha$ on the desired therapeutic outcome.

\section{Stromal cell-derived factor- I $\alpha$}

SDF-1 $\alpha$, also known as CXCL12, is a chemokine produced by stromal fibroblasts, which mediates inflammation and the immune response through modulating lymphocyte chemotaxis (Table 1) (Bleul et al 1996). Additionally, SDF-1 $\alpha$ regulates hematopoeisis and has a role in tumor metastasis (Table 1) (Bleul et al 1996). Like IL-1 $\alpha / \beta$ and TNF $\alpha$, SDF- $1 \alpha$ is ubiquitously found throughout the body, and is an important soluble mediator of the tissue microenvironment.

Within the BM, the primary lineage-specific progeny of MSCs are stromal fibroblasts (Deans and Moseley 2000; Bianco et al 2001). It is not surprising then that SDF- $1 \alpha$ is important in the biology of MSCs. Expression of the principal SDF-1 $\alpha$ receptor, CXCR4, has been demonstrated on MSCs, where it has been shown to mediate site-directed homing of MSCs in models of tissue engineering (Schantz et al 2007). In the $\mathrm{BM}, \mathrm{SDF}-1 \alpha$ is vital to the hematopoietic supportive function that MSCs exert to maintain proper hematopoeisis (Van Overstraeten et al 2006).

As mentioned earlier, preconditioning MSCs with cytokines such as IL-1 $\beta$ and TNF $\alpha$ increased the migratory capacity of the cells (Ponte et al 2007; Schmal et al 2007). However, these enhancing effects were shown to be independent of SDF- $1 \alpha$, and were instead mediated by other chemokines (Ponte et al 2007).

In general, the clinical relevance of SDF-1 $\alpha$ in the success of MSC therapies is positive, since SDF- $1 \alpha$ gradients help MSCs home to sites of tissue injury (Bleul et al 1996). In theory, MSCs could be administered systemically and allowed to respond to SDF-1 $\alpha$ gradients for proper delivery to the target tissue. Once at the site of injury, MSCs themselves could serve as a source of SDF-1 $\alpha$ (Zhang et al 2007). Expression of SDF- $1 \alpha$ by MSCs has been shown to promote survival of cardiac myocytes after myocardial infarction in rats (Zhang et al 2007).

However, SDF- $1 \alpha$ is also a potent lymphocyte chemoattractant (Bleul et al 1996). Excess production of SDF-1 $\alpha$ 
within the microenvironment could potentially lead to increased immune cell infiltration and transplant rejection. SDF-1 $\alpha$ may be less beneficial or even deleterious in therapies transplanting cells partly or fully differentiated from MSCs. For instance, SDF-1 $\alpha$ has been shown to increase the proliferation of neural progenitor cells dissociated from rat cortex (Gong et al 2006). Comparing these results to the example of MSC neuronal transdifferentiation discussed throughout this review, excess SDF-1 $\alpha$ might impede the final steps of neuronal maturation necessary for therapeutic improvement.

To counteract any negative influences of local microenvironmental SDF-1 $\alpha$ on MSC therapies, there are several available pharmacologics that inhibit the SDF-1 $\alpha$ / CXCR4 interaction. Plerixafor, also known as Mozobil ${ }^{\circledR}$ or AMD3100, is a partial antagonist of CXCR4, which has recently competed Phase 3 clinical trials, but is not yet in routine clinical use (Table 1) (Cashen et al 2007). Current in vivo studies have shown that pre-treatment of MSCs with AMD3100 significantly prevented stem cell migration to the injured rat brain (Wang et al 2007). Similar approaches may be beneficial in order to prevent non-specific MSC migration. T134, a small molecule CXCR4 inhibitor, and tannic acid, a water-soluble polyphenol widely distributed within the plant kingdom that acts as a selective CXCR4 antagonist, both inhibit the SDF-1 $\alpha / \mathrm{CXCR} 4$ interaction and may have future application as approved pharmacologics (Table 1) (Arakaki et al 1999; Chen et al 2003). Administration of these compounds would most likely have to be locally delivered to the target tissue, since systemic inhibition of SDF-1 $\alpha /$ CXCR 4 could disrupt BM homeostasis or lead to excessive HSC mobilization into the peripheral circulation.

\section{Summary}

In this review, we address the concept of the tissue microenvironment, and examine its clinical importance in MSC therapies. By accurately developing in vitro models that mimic the local tissue milieu, the behavior of MSCs or their differentiated progeny can be observed prior to in vivo application. Through like approaches, ill effects from soluble mediators or other cell types, which can impede the desired therapeutic outcome, can be assessed. In many cases, co-therapy with a pharmacologic such as a cytokine receptor antagonist may negate the deleterious effects of the microenvironment and optimize the therapeutic potential of MSCs. Here, we have focused on three ubiquitous microenvironmental factors with known effects on MSC function, and addressed how local delivery of inhibitors to these factors could improve the MSC therapy.

\section{References}

Arakaki R, Tamamura H, Premanathan M, et al. 1999. T134, a small-molecule CXCR4 inhibitor, has no cross-drug resistance with AMD3100, a CXCR4 antagonist with a different structure. J Virol, 73:1719-23.

Arend WP. 1993. Interleukin-1 receptor antagonist. Adv Immunol, 54:167-227.

Bianco P, Riminucci M, Gronthos S, et al. 2001. Bone marrow stromal stem cells: nature, biology, and potential applications. Stem Cells, 19:180-92.

Bleul CC, Fuhlbrigge RC, Casasnovas JM, et al. 1996. A highly efficacious lymphocyte chemoattractant, stromal cell-derived factor 1 (SDF-1). J Exp Med, 184:1101-9.

Bresnihan B, Cunnane G. 1998. Interleukin-1 receptor antagonist. Rheum Dis Clin North Amer, 24:615-28.

Cashen A, Nervi B, DiPersio J. 2007. AMD3100: CXCR4 antagonist and rapid stem cell-mobilizing agent. Future Oncol, 3:19-27.

Chen X, Beutler JA, McCloud TG, et al. 2003. Tannic acid is an inhibitor of CXCL12 (SDF-1alpha)/CXCR4 with antiangiogenic activity. Clin Cancer Res, 9:3115-23.

Cheng N, Janumyan YM, Didion L, et al. 2004. Bcl-2 inhibition of $\mathrm{T}$-cell proliferation is related to prolonged T-cell survival. Oncogene, 23:3770-80.

Cho KJ, Trzaska KA, Greco SJ, et al. 2005. Neurons derived from human mesenchymal stem cells show synaptic transmission and can be induced to produce the neurotransmitter substance P by interleukin-1 alpha. Stem Cells, 23:383-91.

Choi KS, Shin JS, Lee JJ, et al. 2005. In vitro trans-differentiation of rat mesenchymal cells into insulin-producing cells by rat pancreatic extract. Biochem Biophys Res Commun, 330:1299-305.

Choy EH, Panayi GS. 2001. Cytokine pathways and joint inflammation in rheumatoid arthritis. N Engl J Med, 344:907-16.

Deans RJ, Moseley AB. 2000. Mesenchymal stem cells: biology and potential clinical uses. Exp Hematol, 28:875-84.

Dinarello CA. 1994. The interleukin-1 family: 10 years of discovery. FASEB J, 8:1314-25.

Eberhardt M, Salmon P, von Mach MA, et al. 2006. Multipotential nestin and Isl-1 positive mesenchymal stem cells isolated from human pancreatic islets. Biochem Biophys Res Commun, 345:1167-76.

Gabay C. 2000. IL-1 inhibitors: novel agents in the treatment of rheumatoid arthritis. Expert Opin Invest Drug, 9:113-27.

Gong X, He X, Qi L, et al. 2006. Stromal cell derived factor-1 acutely promotes neural progenitor cell proliferation in vitro by a mechanism involving the ERK1/2 and PI-3K signal pathways. Cell Biol Int, 30:466-71.

Grassel S, Ahmed N. 2007. Influence of cellular microenvironment and paracrine signals on chondrogenic differentiation. Front Biosci, 12:4946-56.

Greco SJ, Corcoran KE, Cho KJ, et al. 2004. Tachykinins in the emerging immune system: relevance to bone marrow homeostasis and maintenance of hematopoietic stem cells. Front Biosci, 9:1782-93.

Greco SJ, Liu K, Rameshwar P. 2007. Functional similarities among genes regulated by OCT4 in human mesenchymal and embryonic stem cells. Stem Cells, 25:3143-54.

Greco SJ, Rameshwar P. 2007. Enhancing effect of IL-1alpha on neurogenesis from adult human mesenchymal stem cells: implication for inflammatory mediators in regenerative medicine. J Immunol, 179:3342-50.

Greco SJ, Zhou C, Ye JH, et al. 2007. An interdisciplinary approach and characterization of neuronal cells transdifferentiated from human mesenchymal stem cells. Stem Cells Dev, 16:811-26.

Hannum CH, Wilcox CJ, Arend WP, et al. 1990. Interleukin-1 receptor antagonist activity of a human interleukin-1 inhibitor. Nature, 343:336-40. 
Jeon SJ, Oshima K, Heller S, et al. 2007. Bone marrow mesenchymal stem cells are progenitors in vitro for inner ear hair cells. Mol Cell Neurosci, 34:59-68.

Knight DM, Trinh H, Le J, et al. 1993. Construction and initial characterization of a mouse-human chimeric anti-TNF antibody. Mol Immunol, 30:1443-53.

Lerou PH, Daley GQ. 2005. Therapeutic potential of embryonic stem cells. Blood Rev, 19:321-31.

Locksley RM, Killeen N, Lenardo MJ. 2001. The TNF and TNF receptor superfamilies: integrating mammalian biology. Cell, 104:487-501.

Ong SY, Dai H, Leong KW. 2006. Inducing hepatic differentiation of human mesenchymal stem cells in pellet culture. Biomaterials, 27:4087-97.

Ortiz LA, Dutreil M, Fattman C, Pandey AC, Torres G, Go K, Phinney DG. 2007. Interleukin 1 receptor antagonist mediates the antiinflammatory and antifibrotic effect of mesenchymal stem cells during lung injury. Proc Natl Acad Sci U S A, 104:11002-7.

Phinney DG, Prockop DJ. 2007. Concise review: mesenchymal stem/multipotent stromal cells: the state of transdifferentiation and modes of tissue repair - current views. Stem Cells, 25:2896-902.

Ponte AL, Marais E, Gallay N, et al. 2007. The in vitro migration capacity of human bone marrow mesenchymal stem cells: comparison of chemokine and growth factor chemotactic activities. Stem Cells, 25:1737-45.

Potian JA, Aviv H, Ponzio NM, et al. 2003. Veto-like activity of mesenchymal stem cells: functional discrimination between cellular responses to alloantigens and recall antigens. J Immunol, 171:3426-34.
Schantz JT, Chim H, Whiteman M. 2007. Cell guidance in tissue engineering: SDF-1 mediates site-directed homing of mesenchymal stem cells within three-dimensional polycaprolactone scaffolds. Tissue Eng, 13:2615-24.

Schmal H, Niemeyer P, Roesslein M, et al. 2007. Comparison of cellular functionality of human mesenchymal stromal cells and PBMC. Cytotherapy, 9:69-79.

Trzaska KA, Kuzhikandathil EV, Rameshwar P. 2007. Specification of a dopaminergic phenotype from adult human mesenchymal stem cells. Stem Cells, 25:2797-808.

Van Overstraeten-Schlögel N, Beguin Y, Gothot A. 2006. Role of stromal-derived factor-1 in the hematopoietic-supporting activity of human mesenchymal stem cells. Eur J Haematol, 76:488-93.

Wang Y, Deng Y, Zhou GQ. 2007. SDF-1alpha/CXCR4-mediated migration of systemically transplanted bone marrow stromal cells towards ischemic brain lesion in a rat model. Brain Res. in press.

Yan J, Xu L, Welsh AM, et al. 2006. Combined Immunosuppressive Agents or CD4 Antibodies Prolong Survival of Human Neural Stem Cell Grafts and Improve Disease Outcomes in ALS Transgenic Mice. Stem Cells, 24:1976-85.

Zhang M, Mal N, Kiedrowski M, et al. 2007. SDF-1 expression by mesenchymal stem cells results in trophic support of cardiac myocytes after myocardial infarction. FASEB J, 21:3197-207. 
